\title{
Rechtsgeschichte
}

http://www.rg-rechtsgeschichte.de/rg13

Zitiervorschlag: Rechtsgeschichte Rg 13 (2008)

$\mathrm{Rg} 1 \mathbf{3}_{2008}$

$12-24$

http://dx.doi.org/10.12946/rg13/012-024

\section{Caspar Ehlers}

\section{Recht und Raum}

Das Beispiel Sachsens im frühen Mittelalter 


\section{Abstract}

Between the 9th and the 11th centuries the Saxons developed a specific consciousness regarding their freedom; initially related to the Saxon people it came to be associated with the land of the Saxons. This was accompanied by the growth of power held by the Saxons in respect to the other regna of the empire. They were able to boycott royal elections from the year 1002 onwards and thus force the newly elected king to travel to Saxony in order to win their approval in their territory. In the second half of the 11 th century the Saxons endeavoured to make use of the »Saxon freedom « as a right of opposition against the Salian Kings, which precipitated a war with Henry IV, although he was simply trying to establish his own rights as a king within the eastern parts of Saxony (i. e. the reoccupation of the former Ottonian crown domain in and around the Harz mountains, which Henry had inherited). For that reason the war was limited to the eastern parts of Saxony and Thuringia in addition to the lands of the opposition in southern Germany, while Westphalia, for instance, was not touched. Nevertheless, the conflict has been known until our own times as »Henry's IV war with the Saxons«. This is the result of writing history in the 11th century.

In an epoque without states, this is a strong indication how the perception of space changed, intensified and led to the concept of territory. Henry IV was not able to successfully meet this challenge and was never able to develop an appropriate response - as did perhaps all his successors on the German throne. 
I Es handelt sich um erste Überlegungen zu einem Versuch des Neuansatzes. Die Problematik selbst ist häufig bearbeitet worden, eine intensive Auseinandersetzung mit dem Forschungsgegenstand kann hier nicht geleistet werden. Vgl. vor allem: WoLfGANG EgGERT, Das Wir-Gefühl bei fränkischen und deutschen Geschichtsschreibern bis zum Investiturstreit, in: Wir-Gefühl und Regnum Saxonum bei frühmittelalterlichen Geschichtsschreibern, hg. von WOLFGANG EgGERT und BARBARA Pätzold, Berlin I984, I7-I79; Wolfgang EgGert, Identifikation und Wir-Gefühl bei mittelalterlichen Geschichtsschreibern bis zum Investiturstreit, in: Philologus I23 (I979) 54-63; JоACHIM ЕHLERS, Das früh- und hochmittelalterliche Sachsen als historische Landschaft, in: Papstgeschichte und Landesgeschichte. Festschrift für Hermann Jakobs zum 65 . Geburtstag, hg. von JOACHIM DAHLhaus und Armin Kohnle, Köln I995, I7-36; JOACHIM EHLERS, Die Sachsenmission als heilsgeschichtliches Ereignis, in: Vita Religiosa im Mittelalter. Festschrift für Kaspar Elm zum 70. Geburtstag, hg. von Franz J. Felten und NiKOLAS JASPERT, Berlin I999, 37-53; Joachim Ehlers, Sachsen. Raumbewußtsein und Raumerfahrung in einer neuen Zentrallandschaft des Reiches, in: Ottonische Neuanfänge. Symposion zur Ausstellung »Otto der Große, Magdeburg und Europa «, hg. von BERND SCHNEIDMÜLLER und STEFan WeINFURTER, Mainz 200I, 37-57; Wolfgang Giese, Der Stamm der Sachsen und das Reich in ottonischer und salischer Zeit, Wiesbaden I979; H. NowaK, Untersuchungen zum Gebrauch der Begriffe populus, gens und natio bei Adam von Bremen und Helmold von Bosau, Diss. Münster I97I; BERND SCHNEIDMÜLLER, Nomen gentis. Nations- und $\mathrm{Na}-$ menbildung im nachkarolingischen Europa, in: Nomen et Gens. Zur historischen Aussagekraft frühmittelalterlicher Personennamen, hg. von Dieter Geuenich, Wolfgang Haubrichs und Jörg JARnut, Berlin, New York I997,

\title{
Recht und Raum
}

\author{
Das Beispiel Sachsens im früheren Mittelalter
}

Die folgenden Überlegungen gelten dem Zusammenwachsen von räumlichen und rechtlichen Vorstellungen im frühmittelalterlichen Sachsen. Es soll dabei weniger um die Übertragung fränkischer Konzeptionen gehen als darum, wie die Sachsen selbst als Folge ihrer Integration in das christliche Reich eine Vorstellung entwickelten, den ihnen eigenen Raum mit einem Recht zu verbinden, das selbst Ergebnis dieser Niederlage war. Um diesen Prozess zu verfolgen, ist es nötig, auf die wichtigsten Interpretationen der Unterwerfung Sachsens durch die Historiographie des 9. Jahrhunderts zu schauen.

Die im Gebiet des späteren regnum Saxonicum - des Herzogtums - lebenden Menschen und Personengruppen konnten in vorfränkischer Zeit keine gentile Einheit bilden. Erst mit den Sachsenkriegen und vor allem seit der Mission beziehungsweise der Integration der Unterworfenen in das karolingische Reich konnten überhaupt jene Entwicklungen grundgelegt werden, die man rückblickend als »Erfindung Sachsens « bezeichnen könnte. Daher sind die Grundsteine für die historiographisch/literarische Schöpfung eines sächsischen Bewusstseins und eines gleichermaßen daraus hervorgehenden Rechts als Ausdruck eines sächsischen Selbstverständnisses ebenfalls erst ab dem Anfang des 9. Jahrhunderts zu legen gewesen. Und zwar als Ergebnis der Niederlage gegen die Franken, wie schon anderenorts gezeigt wurde. ${ }^{\mathbf{I}}$

In der Vita Karls des Großen ist erstmals der Gedanke eines Aufgehens des sächsischen im fränkischen Volk formuliert, als Einhard († 840) zeitgenössisch über die fränkischen Konzessionen für einen Frieden mit den Sachsen berichtet: »Unter der vom König gestellten und von den Sachsen angenommen Bedingung nahm der Krieg ein Ende, der sich so viele Jahre hingezogen hatte, dass sie dem heidnischen Götzendienst und den heimischen Religionsgebräuchen entsagten, die Sakramente des christlichen Glaubens annähmen und mit den Franken zu einem Volke sich verbänden. « Dieser Gedanke wird in der gut anderthalb Jahrhunderte später entstandenen und gleichfalls berühmten Formulierung Widukinds von Corvey entscheidend abgeschwächt, wie Karl Leyser betont hat. ${ }^{3}$ Widukind († nach 973) sagt nämlich über diese Ereignisse:

I40-I 56; J. SzÜCs, Nation und Geschichte: Studien, Köln, Wien I98I.

2 Eaque conditione a rege proposita et ab illis suscepta tractum per tot annos bellum constat esse finitum, ut, abiecto daemonum cultu et relictis patriis caerimoniis, Christianae fidei atque religionis sacramenta susciperent et Francis adunati unus cum eis populus efficerentur. Einhardi Vita Karoli
Magni, hg. von Oswald HolderEgGer, Hannover I9I I, Nachdruck I965, cap 7. IO.

3 KARL Leyser, Von der sächsischen Freiheit zur Freiheit Sachsens, in: Die abendländische Freiheit vom IO. zum I4. Jahrhundert. Der Wirkungszusammenhang von Idee und Wirklichkeit im europäischen Vergleich, hg. von JoHANNES FRIED, Sigmaringen I991, 67-83, hier 68. 
»Teils durch sanfte Überredung, teils durch kriegerischen Angriff, [be]zwang er [die Sachsen], und erreichte endlich im dreißigsten Jahre seines Kaisertums [...], wonach er so lange Jahre unablässig gestrebt hatte: dass die, welche einst Bundesgenossen und Freunde der Franken waren, nun Brüder und, wie wir jetzt sehen, gleichsam eine gens durch den christlichen Glauben wurden. $\ll^{4}$

Der Unterschied liegt hauptsächlich darin, dass Einhard von einer völligen Unterwerfung und Widukind von einer Integration unter Gleichen, also keiner Verschmelzung, ${ }^{5}$ ausgeht. Bei Einhard ist die Christianisierung weniger Voraussetzung als vielmehr Bedingung für das Geschehene, während Widukind das enge Zusammenkommen beider gentes durch die sächsische Annahme des Christentums betont, denn schließlich seien sie in ferneren Tagen bereits »Bundesgenossen und Freunde« gewesen.

Dass Widukind wesentlich mehr über die Integration der Sachsen in das fränkische Reich sagen konnte als Einhard, braucht nicht betont zu werden. Der Corveyer Mönch fühlte sich dem ottonischen Herrscherhaus so nah wie vielleicht Einhard Karl dem Großen. Und wenngleich Widukind nicht dessen unmittelbare Königsnähe aufweisen konnte, so dürften doch viele seiner Formulierungen die Vorstellungen, etwa von ihrer Herkunft, ${ }^{6}$ und die Absichten Ottos des Großen und seines Umfeldes widerspiegeln.

Der zwischen Einhard und Widukind in den 8oer Jahren des 9. Jahrhunderts schreibende anonyme Poeta Saxo - zu einer Zeit also, in der die Integration Sachsens in das ostfränkische Reich weit fortgeschritten war - greift Einhards Darstellung auf, erweitert sie aber um einen entscheidenden Punkt: dass nämlich Karl der Große den Sachsen bei abschließenden Friedensverhandlungen im Jahre 803 ihre Gesetze zugestanden und von einem Tribut abgesehen habe ut gens et populus fieret concorditer unus. ${ }^{7}$ Der Nachteil dabei ist jedoch, dass dies wohl frei erfunden ist, ${ }^{8}$ auch wenn die nach dem Jahr Ioo8 begonnenen Quedlinburger Annalen erneut den Bericht des Poeta, stark gerafft allerdings, tradieren: Saxones antiqua libertate donavit, heißt es dort. ${ }^{9}$ Der Poeta ist in seiner Kausalität für die Integration näher an Einhard, wenn bei letzterem die Niederlage und bei dem sächsischen Dichter die Übertragung eines Rechtes durch den Sieger ${ }^{10}$ als Grundlage für eine erfolgreiche Eingliederung gesehen werden. Widukind dagegen hebt auf die Akzeptierung einer übergesetzlichen Moralvorstellung ab. Das erlaubt andererseits den Sachsen, eigene Rechts- und christlich

4 Et nunc blanda suasione, nunc bellorum inpetu ad id cogebat, tandemque tricesimo imperii sui anno obtinuit - imperator quippe ex rege creatus est -, quod multis temporibus elaborando non defecit: ob id qui olim socii et amici erant Francorum, iam fratres et quasi una gens ex Christiana fide, veluti modo videmus, facta est. Widukind von Corvey I/I 5 , in: Widukindi monachi Corbeiensis
Rerum Gestarum Saxonicarum libri III, hg. von PAUL Hirsch und Hans-Eberhard Lohmann, Die Sachsengeschichte des Widukind von Korvei, I935, Nachdruck I989, 25. Vgl. immer noch HeLMUT BeUmanN, Widukind von

Korvei. Untersuchungen zur Geschichtsschreibung und Ideengeschichte des Io. Jahrhunderts, Weimar I950.

5 LEYSER, Freiheit (Fn. 3) 68.
6 Vgl. JaceK BanaszKiewicz, Widukind on the Saxon Origins, in: Acta Poloniae Historica 9I (2005) 25-54. Siehe zu Widukinds intellektuellem Horizont auch BERND SCHNEIDMüllER, Widukind von Corvey, Richer von Reims und der Wandel politischen Bewusstseins im Io. Jahrhundert, in: Beiträge zur mittelalterlichen Reichs- und Nationsbildung in Deutschland und Frankreich, hg. von CARLRICHARD BRÜHL (†) und BERND SCHNEIDMÜLlER, München I997, 83-IO2, vor allem $87 \mathrm{f}$. sowie 9093 mit den Nachweisen der älteren Literatur.

7 Poeta Saxo, Annalium de gestis Caroli Magni imperatoris libri quinque, hg. von Paul von WINTERFELD, in: Monumenta Germaniae Historica, Poetae Latini IV/I, Hannover I 899, I-7I, hier $48 \mathrm{f}$.

8 Dieses nur bei dem so genannten Poeta Saxo, einem anonymen Dichter vielleicht aus dem Corvey der Zeit Arnulfs, überlieferte Ereignis (Die Regesten des Kaiserreiches unter den Karolingern 75 I-9I 8, neubearbeitet von E. MüHLBACHER, vollendet von J. LECHNER, Innsbruck ${ }^{2}$ I908, Nr. 398b) wird im Allgemeinen angezweifelt seit BERNHARD vON Simson. Der Poeta Saxo und der angebliche Friedensschluss Karls des Grossen mit den Sachsen, in: Neues Archiv der Gesellschaft für ältere deutsche Geschichtskunde 32 (I907) 27-50; vgl. ECKHARD Freise, Das Frühmittelalter bis zum Vertrag von Verdun, in: Westfälische Geschichte I, hg. von Wilhelm Kohl, Düsseldorf I983, 275-336, hier 303 .

9 Annales Quedlinburgenses, hg. von Martina Giese, 2004, 437. Vgl. zu den frühen sächsischen Rechtsaufzeichnungen zusammenfassend Karl Kroeschell, recht und unrecht der saßen. Rechtsgeschichte Niedersachsens. Göttingen 2005, I6-27.

Io Vgl. zu der Spannung zwischen Argumenten und Gewalt in der sächsischen Geschichtsschreibung vor allem des 9. Jahrhunderts Helmut Beumann, Die Hagiographie »bewältigt «. Unterwerfung und Christianisierung der Sachsen durch Karl den Großen, in: Settimane di studio del Centro 
italiano di studi sull'alto medioevo 28 (I982) I 29-I68, hier I49I63, mit den an dieser Stelle nicht weiter erörterten historiographischen Beispielen.

i I Matthias Becher, Volksbildung und Herzogtum in Sachsen während des 9. und Io. Jahrhunderts, in: Mitteilungen des Instituts für Österreichische Geschichtsschreibung I08 (2000) 67-84, vor allem $74 \mathrm{f}$.

I2 Vgl. Matthias Becher, Zwischen König und »Herzog «. Sachsen unter Kaiser Arnolf, in: Kaiser Arnolf. Das ostfränkische Reich am Ende des 9. Jahrhun-

derts, hg. von Franz Fuchs, กั่ München 2002, 89-I2I.

begründete Ordnungskonzepte zu entwickeln; dass sie dies auch unternahmen, wird im Folgenden dargelegt werden, dass dies überhaupt nur möglich war, weil sie zuvor von den Franken unterworfen und integriert worden waren, hat Matthias Becher unlängst hervorgehoben. ${ }^{\text {II }}$

Auch wenn heutzutage der Wahrheitsgehalt der Imagination des sächsischen Poeten zu bestreiten ist, so bleibt doch das Bild von Karl als dem Stifter sächsischen Rechts, welches bis über das I I. Jahrhundert hinaus wirksam bleibt, wie wir sehen werden. Darüber hinaus ist festzustellen, dass der Anonymus am Ausgang des 9. Jahrhunderts, in einer krisenhaften Zeit des ostfränkischen Karolingerreiches also, ${ }^{\mathrm{I2}}$ in seiner Darstellung Freiheit und Gesetz $^{13}$ zusammenführt und dies dann mit der gens der Sachsen verknüpft. Also bezieht sich der erfundene Erlass beim Poeten noch nicht auf einen Raum, sondern auf einen Verband - wie es schon Einhard verstand -, damit dieser mit den Franken zu einem »Volk « verschmelzen könne, wie es dann von Widukind später dargestellt werden wird, der jedoch die fides Christiana und nicht die lex Saxonum oder gar eine sächsische libertas als Grund für die Integration begreift. In der substantivischen Form kommt »Libertas « bei Widukind von Corvey überhaupt nur einmal im Kontext der Ursprungssagen vor - und dies in keinem auf die Sachsen bezogenen,$-{ }^{14}$ daneben gibt es nur noch zwei weitere Belege ${ }^{15}$ in anderem Zusammenhang als sächsischem, so dass festgestellt werden kann, dass für den Corveyer Mönch die sächsische Freiheit weniger ein Thema gewesen ist. Eher registrierte Widukind, dass die Sachsen »frei « herrschen, selbst Tribute auferlegen und somit als freies Volk andere zu Unfreien machen konnten. ${ }^{16}$

Dennoch ist die Verbindung von libertas und patria beim Poeta Saxo nicht einzigartig, denn schon in der etwa eine Generation zuvor entstandenen » Translatio sancti Alexandri« wird eine $\mathrm{Zu}$ sammenführung beider Begriffe vorgenommen: pro libertate ac patria ${ }^{17}$ Allerdings wäre zu fragen, inwieweit »Patria " hier als »Heimat« oder als »Vaterland « zu übersetzen wäre, ob also ein kleinerer, ein größerer oder überhaupt kein konkreter Raum dem Autor vor Augen stand, denn er verwendet beide Termini in einem speziellen Kontext, nämlich dem der vorchristlichen Frühgeschichte der Sachsen. Diese endet - wie überhaupt der sächsische Widerstand gegen die Franken - für den Verfasser aus der Mitte des 9. Jahrhunderts mit der Taufe Widukinds in Attigny im Jahre 785,

I3 Poeta Saxo (Fn. 7) 48, Zeilen I I ff.: Tum sub iudicibus [...] legatisque suis permissi legibus uti Saxones patriis et libertatis honore.

I4 Widukind (Fn. 4) I/9, I 2.

I5 Widukind (Fn. 4) II/2O und III/53.

I6 LEYSER, Freiheit (Fn. 3) 72 f.

I7 Translatio sancti Alexandri, hg. von Georg Heinrich Pertz, in: MGH Scriptores 2, I 829, Nachdruck I976, 673-68I, hier cap. I
(675/Z. I) im Zusammenhang mit der sagenhaften Gründungsüberlieferung der Sachsen; vgl. ThоMAS EICHENBERger, Patria.

Studien zur Bedeutung des Wortes im Mittelalter, Sigmaringen I99I, I64 f. mit Anm. 533. Siehe aber auch die Kapitel 2 und 3 der Translatio sancti Alexandri (S. 675 ff.) zu den Gesetzen und Gebräuchen der Sachsen sowie ihrer Bekehrung von der Irrlehre. 
also gut zwei Jahrzehnte früher als das tatsächliche Ende der Sachsenkriege. ${ }^{\text {I8 }}$

Dass der sächsische Diskurs über ihre Freiheit offenbar das ganze Io. Jahrhundert angedauert hat, ist schon daraus abzuleiten, dass Thietmar von Merseburg († IоI8) gleich zu Beginn seiner Chronik die Gefahr beklagt, welche für die (sächsische) Freiheit durch einen schwachen Herrscher ausgeht; für ihn war nämlich, wie Karl Leyser resümierte, »diese libertas identisch mit den Vorteilen und Privilegien, die das sächsische Volk genoss, aus dem die Königsfamilie stammte ${ }^{\text {I }}$ 9

Darüber hinaus lässt sich in Thietmars Chronik aus den ersten Jahren des I I. Jahrhunderts ein sehr differenzierter Freiheitsbegriff erkennen, der neben der Befreiung von Tributen und anderen Lasten als äußeren Kennzeichen auch zwischen der Freiheit des Herrschers und der des `Volkes zu unterscheiden in der Lage ist. ${ }^{20}$ Thietmars Bezug ist demnach auch die Gens, und zwar besonders die den König stellende, die Sachsen. Daneben hat er einen »vielschichtigen politischen patria-Begriff « entwickelt, wie Thomas Eichenberger aufgezeigt hat. ${ }^{2 \mathrm{I}}$

Die Beurteilung des Begriffes patria im sächsischen Kontext

Thomas Eichenberger hat in seiner breit angelegten Studie über den Terminus der Patria vom 6. bis zum I2. Jahrhundert selbstverständlich auch und vor allem sächsische Beispiele herangezogen. ${ }^{22}$ Allerdings ist der Terminus bei Eichenberger von vorneherein stark saufgeladen<, da der Verfasser die Verwendung des Begriffes als Indikator für das wertet, was in unserer vorliegenden Studie als gestreckter Prozess einer Identitätsstiftung untersucht wird. Weil Eichenberger stets vom »verfassungsgeschichtlichen « Kontext des Begriffes spricht, ${ }^{23}$ steht die interpretierende Übersetzung von »Patria « zu Beginn der Untersuchung fest, die Bedeutungsentwicklung hingegen wird nur im Rahmen der rechtsbeziehungsweise verfassungsgeschichtlichen Prämisse nachgezeichnet. $^{24}$

Widukind verwendet den Begriff zwar an herausgehobener Stelle, etwa wenn er den ottonischen Herrschern Heinrich I. und Otto dem Großen im Zusammenhang mit den Siegen über die Ungarn 933 und 955 jeweils den schmückenden, antiken Titel

I8 Translatio sancti Alexandri (Fn. I7), cap. 4 (676).

I9 Die Chronik Thietmars von Merseburg und ihre Korveier Überarbeitung, hg. von Robert HolTzMANN, München I980, hier I/I9, 24 ff., vgl. LeYser, Freiheit (Fn. 3) 73.

20 LEYSER, Freiheit (Fn. 3) 74.

2 I Eichenberger, Patria (Fn. I7) Zitat auf I49.
22 Ebenda, vor allem 66-70, I49-

I 5 5, I 59-I 73 (zu den sächsischen Konflikten Heinrichs IV. sowie zur "Dualität " von ecclesia und patria, vgl. dazu unten).

23 Ebenda $236 \mathrm{ff}$. in der Zusammenfassung und öfters im Haupttext. 24 Ebenda $238 \mathrm{ff}$. 
25 Eichenberger, Patria (Fn. I7) I 50 ff. Vgl. zu Heinrichs I. Königtum nun Wolfgang Giese, Heinrich I. Begründer der ottonischen Herrschaft, Darmstadt 2008.

26 Thietmar (Fn. 19) hier II/45, 92/94 (Codex I) bzw. 93/95 (Codex 2). Thietmar stand der »ottonischen Hausüberlieferung « übrigens fern, vgl. Helmut Lippelt, Thietmar von Merseburg. Reichsbischof und Chronist, Köln 1973, I77I 85 . Insgesamt zu diesem Problemfeld nun Martina GIESE, Die Historiographie im Umfeld des ottonischen Hofes, in: Die Hofgeschichtsschreibung im mittelalterlichen Europa. Projekte und Forschungsprobleme, hg. von Rudolf SCHIEFFER und Jarostaw Wenta, Torún 2006, I9-37, vor allem $26 \mathrm{f}$.

27 Eichenberger, Patria (Fn. I7) I $5 \circ \mathrm{f}$.

ֻั.

28 Ebenda 66-70; Bernd SCHNEIDMÜlleR, Nomen Patriae. Die Ent-

pater patriae zuerkennt. Ob es sich dabei um "Lesefrüchte " des Corveyer Mönches gehandelt hat, ist in der Forschung umstritten, wird aber von Eichenberger abgelehnt. ${ }^{25}$

Ihm geht es in der Interpretation aber weniger um »Patria « selbst als um die darauf bezogenen Personen: Thietmars Formulierung von Otto I. als defensor patriae nach Karl dem Großen beispielsweise ${ }^{26}$ wird erst signifikant durch den »Defensor " ${ }^{27}$ der Ehrentitel für die Bezwinger der Ungarn erst durch »Pater « und so fort. Eine Interpretation des Begriffes von der »Patria « ohne seine rückgebunden-erklärende Verwendung als Beiwort ergäbe übrigens, so Eichenberger, doch eher eine Verwendung im Sinne von "Heimat ", also dem Raum der eigenen Herkunft, in deutlicher Absetzung zum politischen Begriff vom »Regnum $« .{ }^{28}$ Beachtenswert sind die Beobachtungen Eichenbergers zum christlichen Bedeutungsfeld: "Patria « caelestis, aeterna, superna oder divina. Denn diese scheinen überwiegend - mit Ausnahme von superna bei Petrus Damiani - aus dem 9. und Io. Jahrhundert zu stammen. ${ }^{29}$ Eine Spanne, an deren Ende Fulbert von Chartres († I028) die Definition vom Reich als Dreiklang aus Land (terra/patria), Volk (populus) und König (persona regis electi) entwickelt hat, ohne die es kein Reich geben kann (regnum esse non possit). ${ }^{30} \mathrm{Zudem}$ fällt in diese Zeit ab etwa den späten Jahren Ottos I. die zaghafte Entstehung einer Historiographie im engeren Umfeld der ottonischen Herrscher. ${ }^{3 \mathbf{r}}$

\section{Die beginnende Salierzeit: Königliches Ausgreifen nach Sachsen}

Die von Thietmar († IOI 8) gefürchtete Situation trat IO24 tatsächlich ein: Der nächste König kam nicht aus Sachsen. Konrad II., der salische Nachfolger des letzten Ottonen, wurde ohne die Beteiligung der Sachsen in Kamba gewählt. ${ }^{32}$ Allerdings war dies zwei Jahrzehnte zuvor schon Heinrich II. widerfahren, der ebenfalls zu den Sachsen in das Land kommen musste, um sich gleichsam nachwählen zu lassen. I0O2 waren Kirchberg in Thüringen und Merseburg in Ostsachsen, I024 hingegen Minden in Westsachsen die jeweiligen Orte. Bemerkenswert ist dabei die Charakterisierung der sächsischen Gesetze als lex crudelissima durch den Burgunder Wipo, als er von Konrads II. Anerkennung durch die Sachsen be-

stehung Frankreichs in der politisch-geographischen Terminologie (I0.-I3. Jahrhundert), Sigmaringen 1987 , hier $53 \mathrm{ff}$. stellt ebenfalls patria als »unpolitische Raumbezeichnung « vor.

29 In der Zusammenfassung bei Eichenberger, Patria (Fn. I7) 237.

30 Fulbert von Chartres, Tractatus contra Iudaeos, in: MPL I4I, col. 305-3 I 8, hier col. 307; vgl.
SCHNeIdmüller, Nomen Patriae (Fn. 28) $54 \mathrm{f}$.

3 I Vgl. Giese, Historiographie (Fn. 26) 22-25.

32 Siehe nun Jörg Rogge, Die deutschen Könige im Mittelalter. Wahl und Krönung, Darmstadt 2006, I3-I7, der die bestätigende Nachwahl in Minden nicht eigens hervorhebt. 
richtet, deren Voraussetzung die Anerkennung ebendieser Rechte gewesen sei. ${ }^{33}$

Hier wäre eine neue Stufe zu konstatieren: Der Raum Sachsens wird verdeutlicht durch den Zwang für die Herrscher, zur Krönung in ihn zu reisen, während die Freiheiten und das Recht des Verbandes zum einen durch sein Fernbleiben bei einer Wahl außerhalb sowie durch die Bestätigung seiner »Gewohnheiten « im Zuge der Nachwahl manifestiert werden. ${ }^{34}$ Eine Trias Freiheit-Recht-Raum wird nunmehr für Sachsen erkennbar, die offenbar seit der Wende des 9. zum Io. Jahrhundert konstruiert und durch die ottonische Königsherrschaft verfestigt wurde. Dieses Bewusstsein wird während der Regierungszeit Heinrichs IV. schließlich ungeschminkt zutage treten.

Zusammengefasst lässt sich für Sachsen feststellen, dass im ersten Viertel des I I. Jahrhunderts ein ursprünglich gentiler Freiheitsbegriff auch auf einen Raum bezogen und dies symbolisch zum Ausdruck gebracht wird, indem der König zu den Fernbleibenden reisen muss. Das tut er erstmals 1002 (Heinrich II.) und erneut I024 (Konrad II.). Sowohl wegen der Wiederholung dieses Vorgehens und auch wegen der Beobachtung, dass Heinrich III. nach seiner Krönung am I4. April I028 in Aachen zuerst mit seinem Vater durch Westfalen nach Ostsachsen zog, ${ }^{35}$ kann von einem vom Königtum nolens volens respektierten Anspruch der Sachsen auf ein derartiges Procedere ausgegangen werden.

Vorgreifend ist anzumerken, dass es dann unter Heinrich III. bereits Bestrebungen sächsischer Adeliger geben hat, sich von der Herrschaft des Saliers gewaltsam zu befreien, ${ }^{36}$ und dass sich auch in anderen Regionen seines Reiches Aufstandskeime mit regionalen Rückbezügen, etwa in Lothringen oder Bayern, entwickelt hatten. ${ }^{37}$ Allerdings ist auch zu betonen, dass es kaum einen ostfränkisch-deutschen König gegeben hat, der keine Schwierigkeiten mit Sachsen hatte. ${ }^{38}$

\section{Das spätere II. Jahrhundert: Das Land der Freien}

Im Zuge des I I. Jahrhunderts verfestigte sich ein sächsisches Selbstbewusstsein gegenüber den landfremden salischen Herrschern aus dem Mittelrheingebiet. Angelegt worden dürfte dies sein in einem lang gestreckten Prozess, an dem die Historiographie

33 Wipo, Gesta Chuonradi imperatoris, cap. 6, in: Die Werke Wipos, hg. von Harry Bresslau, I9I 5 , Nachdruck I977, I-62, hier 29. Vgl. auch die überlieferte Kritik der Sachsen an Konrad II. und Heinrich III.: Carmen de bello Saxonico, neu herausgegeben und übersetzt von Franz-JoseF Schmale, in: Quellen zur Geschichte Kaiser Heinrichs IV., bearbeitet von Franz-JoseF
Schmale und Irene OtT-SchmaLE, Darmstadt I963, I43-I89, hier I, 29-50 (I47).

34 Vgl. die Episode mit dem "Schweinezins « aus dem Jahr IOO2, MATTHIAS Werner, Die Anfänge eines Landesbewußtseins in Thüringen, in: Aspekte thüringisch-hessischer Geschichte, hg. von MicHAEL GoCKel, Marburg/Lahn I992, 8I-I37, hier 94-97.

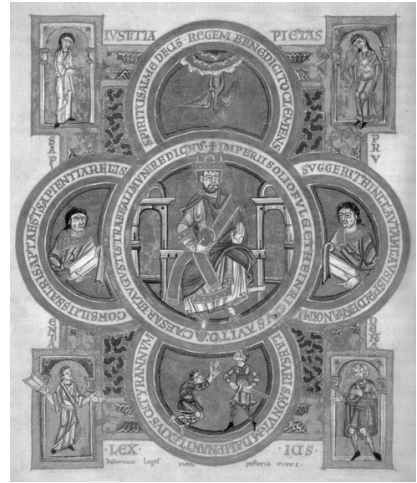

Heinrich II.

35 Aachen-Dortmund-MagdeburgAllstedt-Wallhausen-ImbshausenPöhlde: vgl. Regesta Imperii III. Die Regesten des Kaiserreiches unter den Herrschern aus dem salischen Haus IO24-I I25: I. Abteilung, neubearbeitet von Heinrich Appelt, Graz I95 I, Nr. I I 7 a bis I 35 .

36 Gerd Althoff, Die Billunger in der Salierzeit, in: Die Salier und das Reich, hg. von STEFAN WeINFURTER, Sigmaringen I99I, hier Band I, 309-329, vor allem 3 I9 ff.

37 Vgl. im Überblick: STEFAN WeINFURTER, Ordnungskonfigurationen im Konflikt. Das Beispiel Kaiser Heinrichs III., nun in: DERs., Gelebte Ordnung, gedachte Ordnung. Ausgewählte Beiträge zu König, Kirche und Reich, hg. von Helmut Kluger, Hubertus SeIbert und Werner Bomm. Ostfildern 2005, 265-287, hier $282 \mathrm{ff}$.

38 Vielleicht nur der Süpplingenburger Lothar III.? Der hatte jedenfalls das Problem, dass die Herzöge von Bayern und Schwaben seiner Wahl ferngeblieben waren und dass mit Konrad (III.) ein Schwabe als Gegenkönig aufgestellt wurde. 
39 Vgl. dazu nun: Matthias BeCHER, Die Auseinandersetzung Heinrichs IV. mit den Sachsen. Freiheitskampf oder Adelsrevolte?, in: Vom Umbruch zur Erneuerung? Das II. und beginnende I2. Jahrhundert. Positionen der Forschung, hg. von JöRG JARNUT

㝘

und MatThias Wemhoff unter Mitarbeit von Nicola Karthaus, München 2006, 357-378.

40 Brunos Buch vom Sachsenkrieg cap. 25, in: Quellen zur Geschichte Kaiser Heinrichs IV. (Fn. 33) $222 \mathrm{ff}$.

4I Dieser Satz ist nach Sallust, Catilina 20,9, formuliert, vgl. C. Sallvsti Crispi Catilina, Ivgvrtha, Fragmenta ampliora, post A. W. Ahlberg ed. Alphonsvs
Kvrfess, 3. Aufl., Leipzig I957. Dazu auch Leyser, Freiheit (Fn. 3) $77 \mathrm{f}$.

42 Dieser Satz ist nach Sallust, Jugurtha 3 I,I I, formuliert, wobei es dort heißt: in imperio nati, was sinngemäß dasselbe bedeutet, nur die soziale Schichtung anders bezeichnet, vgl. Fn. 4I. Zu dieser Problematik siehe ebenfalls LEYSER, Freiheit (Fn. 3) 80 mit weiteren Verweisen. 
er aufgehört hatte, ein König zu sein, war der nicht mehr vorhanden, dem ich Treue zu bewahren hatte. Also nicht gegen den König, sondern gegen den ungerechten Räuber meiner Freiheit; nicht gegen das Vaterland [die Heimat?], sondern für das Vaterland [die Heimat?] und für die Freiheit, welche kein braver Mann anders als mit dem Leben zugleich hingibt, ${ }^{43}$ ergreife ich die Waffen und fordere von Euch, dass auch Ihr sie ergreift. ${ }^{44}$ Erwachet also und bewahret das Erbe, welches Eure Väter Euch hinterlassen haben, für Eure Kinder; hütet Euch, dass nicht durch Eure Sorglosigkeit oder Trägheit Ihr selbst und Eure Kinder fremder Menschen Knechte werdet.《

Inwieweit hier patria tatsächlich mit "Vaterland « oder besser mit » Heimat « zu übersetzen ist, sei hintangestellt. Es ist für unseren Kontext zunächst die »Freiheit« zu beachten, denn sie spielt eine zentrale Rolle in der durch Brunos Buch vom Sachsenkrieg bekannten Argumentation Ottos von Northeim, der salische Burgenbau innerhalb Sachsens diente ihm dabei als Exempel und Anlass. Als althergebracht dargestellte Freiheiten - das »Erbe « - werden in der Rede zum entscheidenden Argument.

Die Ansprache spiegelt in der überlieferten Form kaum Ottos Worte, da die Rede nicht in Latein gehalten wurde. Die Überlieferung ist nämlich an klassischen Vorbildern (wie Sallusts »Coniuratio Catilinae" und "Bellum Iugurthinum «) orientiert, was in Frage stellt, ob Otto in Hötensleben 1073 tatsächlich so gesprochen hat, wie es der gebildete Ostsachse Brun berichtet. ${ }^{45}$ Auf dieses substantiell quellenkritische Problem hat Karl Leyser hingewiesen: »Konnte Otto von Northeim eine solche Rede gehalten haben und konnte er einen allgemeinen, umfassenden Freiheitsbegriff verkünden und von seinen Hörern verstanden werden? Hier stellt sich auch das Problem der sächsischen Landessprache, in der alle diese Ideen ihren Ausdruck finden mussten. «Im Resümee zum Bericht Brunos bemerkt Leyser, dass der angezogene Freiheitsbegriff keine anlassbezogenen oder gar spezifisch sächsischen Problematiken aufweise. ${ }^{46}$ Inwieweit sich die Argumente Ottos überhaupt an untere Schichten des Adels oder gar an die Bauern richtete, muss gefragt werden, denn im Endeffekt entschied und handelte der sächsische Hochadel auf Grund eigenen Rechtes und nicht wegen der Zustimmung des "Volkes «, das in diesem Zusammenhang ohnehin kaum definierbar erscheint, wie Lutz Fenske betonte. ${ }^{47}$ Am Rande ist hervorzuheben, dass gerade

43 Dieser Nebensatz ist wieder nach Sallust, Catilina 33,4, formuliert, vgl. Fn. 4I. Dazu Eichenberger, Patria (Fn. I7) I64-I67, der den Antikenbezug nicht erwähnt, jedoch darauf verweist, dass nicht jeder der sächsischen Opposition reichsweite Bezüge vor Augen hatte. Siehe zu dieser Problematik insgesamt die Studie von ERNST H. KANTOROWICZ, »Pro patria mori « in Medieval Political Thought, in:
American Historical Review 56 (I950/5I) 472-494.

44 Itaque non contra regem, sed contra iniustum meae libertatis ereptorem, non contra patriam, sed pro patria et pro libertate mea, quam nemo bonus nisi cum anima simul amittit, arma capio et, ut vos ea mecum capiatis, expostulo.

45 Brunos Buch vom Sachsenkrieg

(Fn. 40) cap. 24-26, 222-226. Vgl. Leyser, Freiheit (Fn. 3).
46 Leyser, Freiheit (Fn. 3) 78 f. Siehe auch zusammenfassend zur Problematik nun Helmut FlachenECKER, Wider die Tyrannis. Grundlegung des Widerstandsrechts im philosophisch-theologischen Denken des Mittelalters, in: Portraits zur Geschichte des deutschen Widerstands, hg. von MATTHIAS STICKLER, Rahden/Westfalen $2005,25-40$.

47 Lutz Fenske, Adelsopposition und kirchliche Reformbewegung im östlichen Sachsen. Entstehung und Wirkung des sächsischen Widerstands gegen das salische Königtum, Göttingen I977, $57 \mathrm{ff}$. 
Sallust bei den Historiographen recht beliebt gewesen ist, die sich im Zusammenhang mit den Kriegen gegen Heinrich IV. zu Worte melden.

Die Besetzung des Raumes durch Burgen ist für Otto und die Opposition gleichbedeutend mit einer Beschneidung der sächsischen Freiheit. ${ }^{48}$ Der Fall, dass der Herrscher seine eigenen Rechte wahrnimmt, wird durch die Diskussion seiner Rechtmäßigkeit in Frage gestellt und der Diskurs eröffnet, wann man seines Treuegelöbnisses durch Unrecht des Herrschers entbunden wird. Letzteres zielt auf die Personenverbände in der Struktur einer »Herrschaft ohne Staat «(Gerd Althoff), erstes hingegen auf den Raum. So wird Sachsen zu einem »land of the free ", in dem Raum und Menschen gleichermaßen geschützt sind.

Zur gleichen Zeit wird übrigens in der kirchlichen Sphäre der Zusammenhang von libertas, Recht und Raumbezug entwickelt in Form des Klosters und seines Besitzes beispielsweise -, wie Brigitte Szabó-Bechstein herausgearbeitet hat. ${ }^{49}$ Karl Leyser hat im Zusammenhang mit Ottos von Northeim Rede in Hötensleben in gewisser Weise ergänzend darauf aufmerksam gemacht, dass »die Bischöfe [...] von der Freiheit ganz Sachsens, die Laien nur von ihrer eigenen " gesprochen hätten. ${ }^{5 \circ}$ Hier wird der unterschiedliche Blick deutlich, den Kirche und Welt entwickeln. Die Schnittmenge ist schließlich die Beteiligung ostsächsischer Bischöfe an der Erhebung. Es war ohnehin nur der Hochadel Ostsachsens bei der Versammlung in Hötensleben anwesend gewesen, die also keinesfalls als »sächsischer Stammestag " bezeichnet werden darf.

Zusammenfassend kann gesagt werden, dass Otto von Northeim zwar den Begriff der Freiheit angeführt haben dürfte, dass es aber Bruno war, der die literarischen Verknüpfungen erschuf, den Antikenbezug, zu dem Otto wohl nicht fähig war. Das bedeutet wiederum, dass des Northeimers Freiheitsbegriff in einem allgemeinen, gleichwohl doch sächsischen, Selbstverständnis wurzelte, während Bruno auf einen elaborierten Diskurs zurückgriff, der in den intellektuellen Kreisen der Opposition gegen Heinrich IV. geführt wurde. Auch wenn der Graf dabei »auf Grund eigenen Rechtes « handelte (Fenske) oder zu handeln glaubte und sich auch nicht unbedingt »spezifisch sächsischen Problematiken « (Leyser) gegenübersah, ${ }^{{ }^{I}}$ so ist es doch das Bild der Historiographie, mit dem wir zu arbeiten haben. Und dies ist, wie schon bei Widukind und Thietmar beobachtet, eben ausgefeilter und verfügt über einen

48 Zuletzt: Becher, Auseinandersetzung (Fn. 39).

49 Brigitte Szabó-Bechstein, Libertas Ecclesiae. Ein Schlüsselbegriff des Investiturstreits und seine Vorgeschichte, 4.- I I. Jahrhundert, Rom I985, etwa I 23 ff. und öfters. Vgl. schon das fünfte Kapitel »Der Kampf um die rechte Ordnung in der christlichen Welt " bei Gerd Tellenbach, Libertas. Kirche und Weltordnung im Zeit- alter des Investiturstreites, Stuttgart I936, Nachdruck I996, I 5 II92. Siehe auch EICHENBERGER, Patria (Fn. I7) I67 f., der nicht auf die genannte Arbeit Szabó-Bechsteins eingeht.

50 LEYSER, Freiheit (Fn. 3) 79.

5 I Das jedenfalls dürfte ihm klar gewesen sein, denn das Adelsbündnis gegen Heinrich IV. ging ja weit über Sachsen hinaus. 
weiteren Horizont, als ihn vielleicht die Akteure in Hötensleben oder anderswo hatten.

Die im Frieden von Gerstungen zugesagte Schleifung der Harzburg und der übrigen Befestigungen Heinrichs IV. in Ostsachsen $^{52}$ war vermutlich das wichtigste Ziel der ostsächsischen Opposition, die sich ihre zuvor während der Phase der Unmündigkeit Heinrichs IV. angeeigneten Gewohnheiten im Umgang gerade auch mit Reichsgut nicht nehmen lassen wollten. ${ }^{53}$ Mit Hilfe dieser und weiterer Befestigungsanlagen ${ }^{54}$ war schließlich durch Heinrichs geostrategische Initiative ein Ring um den Harz entstanden, dessen Funktion die Sicherung des ausgedehnten wirtschaftlich sowie schon damals historisch höchst bedeutenden Königsgutes in jenem Mittelgebirge gewesen war. Im Unterschied zu seinen Vorgängern war Heinrich IV. nun durch seine Burgen und deren landfremde Besatzungen permanent präsent, auch wenn er sich nicht selbst in Sachsen aufhielt. Im Gegensatz etwa zu den Burgen aus den zwanziger Jahren des Io. Jahrhunderts, die - wie in der Rede Ottos von Northeim in Hötensleben vorgebracht - während der Herrschaft Heinrichs I. dem Schutz vor den äußeren Feinden dienten, richteten sich die Heinrichs IV. unübersehbar nach innen.

Sie zielten auf den Raum, nicht auf den Verband der Sachsen an sich. Der hingegen wird erst zum Ziel des Saliers durch das sowohl bei Lampert von Hersfeld als auch bei Bruno referierte Gerücht, Heinrich IV. hätte die Ausrottung aller Sachsen im Auge gehabt. ${ }^{55} \mathrm{Da}$ es sich bei dieser Darstellung ohne jeden Zweifel um Propaganda handelt, ist leicht festzustellen, dass das Gemunkel notwendig war, um eine Personengruppe zu rechtfertigen und auf ein hohes politisches Niveau zu heben, die bei ihrer Rebellion offenbar nicht das Wohl aller Sachsen vor Augen hatte.

So lässt sich zusammenfassen, dass keine Erhebung des sächsischen »Volkes « im eigentlichen Sinne stattgefunden hat, sondern eine Revolte von Interessengemeinschaften, die durch Angehörige der Führungsschichten geleitet, aber nicht immer kontrolliert wurde. Reichsweite, nicht unbedingt sächsische Bezüge - wie die Kritik an Heinrich III. und Heinrich IV. als schlechten Herrschern zeigt prägen das Geschehen, sie erlauben beispielsweise erst die Mobilisierung und später die Bestrafung der »Bauern« (wegen ihrer unkontrollierten Übergriffe). Der »Sachsenkrieg " gegen Heinrich ist keine Erhebung der »bäuerlichen Bevölkerung «. Instrumentalisierung durch ihre adeligen Herren wäre vielmehr der geeignete

52 Haec autem sunt castella, quae ipse, postquam pater eius decesserat, extruxit, quae tamen ad presens memoriae occurrunt: Hartesburg, Wigantestein, Moseburg, Sassenstein, Spatenberg, Heimenburg, Asenberg. Vokenroht [Volkenrode bei Mühlhausen?] Friderici palatini comitis fuerat, idque ei rex quadam legum violentia eripuerat, presidiumque suum imposuerat. Liuneburc
[Lüneburg] quoque, oppidum maximum Ottonis ducis Saxonici, situm in confinio Saxonum et $\mathrm{Lu}$ ticiorum, occupaverat, militesque lectissimos cum Eberhardo filio Eberhardi comitis de Ellenburc imposuerat [...]. Lampert von Hersfeld = Lamperti monachi Hersfeldensis Opera, hg. von Oswald Holder-EgGer, I 894, Nachdruck I984, I 59; FENSKE,
Adelsopposition (Fn. 47) 28-32; LEYser, Freiheit (Fn. 3) 77.

53 Vgl. dazu demnächst CASPAR EHLERs, Pfalzen und Burgenbau in Sachsen, in: Kolloquiumsband „Die Salier und Sachsen " ed. Goslarer Geschichtsverein. (erscheint 2009).

54 Gerhard Streich, Burg und Kirche während des deutschen Mittelalters. Untersuchungen zur Sakraltopographie von Pfalzen, Burgen und Herrensitzen, Sigmaringen I984, hier Bd. 2, $436 \mathrm{ff}$.

55 Lampert (Fn. 52) ad a. 1073 (I46f.) sowie ad a. I075 (2I2) und (233); Brunos Buch vom Sachsenkrieg (Fn. 40) cap. 20, 2 I 8 . 
56 Nam rex non tantum illis, qui scelus confesse fecerant, quantum istis, qui se a perpetrato scelere purgabant, irascitur; et dedignans irasci in rusticos, in maximos buius regionis homines furorem suum, si quando tempus haberet, accendere meditatur. Brunos Buch vom Sachsenkrieg (Fn. 40) cap. $34,236 \mathrm{ff}$.

57 Lampert von Hersfeld (Fn. 52) 2 I If.

58 Speziell über die Vorwürfe des sexuellen Ausschweifens wäre meines Erachtens noch einmal nachzudenken unter dem Aspekt, inwieweit es sich um Metaphern für die »Vergewaltigung « etwa der Kirche (personifiziert beispielsweise durch die Schwester, die Äbtissin von Quedlinburg) gehandelt haben könnte. GERD Tellenbach, Der Charakter Heinrichs IV. Zugleich ein Versuch über die Erkennbarkeit menschlicher Individualität im hohen Mittelalter, in: Person und Gemeinschaft im Mittelalter. Festschrift für Karl Schmid zum fünfundsechzigsten Geburtstag, hg. von Gerd Althoff, Dieter Geuenich und Otto Gerhard OeXle, Sigmaringen I988, 345367; August Nitschke, Die Ziele Heinrichs IV. Beobachtungen zum Wandel einer Staatsform, in: Wissenschaft, Wirtschaft und Technik. Studien zur Geschichte. Wilhelm Treue zum 60. Geburtstag, hg. von K. H. Manegold, München I969, 38-63.

59 Das Register Gregors VII. (Gregorii VII Registrum), hg. von Erich Caspar, Hannover I920/ I923, Nachdruck I990, hier Brief Nr. III/7 an Heinrich IV. (S. 256259) vom Anfang September

Begriff. Dies wird dadurch unterstrichen, dass Heinrich IV. auch nicht die Bauern (rustici) bestrafen ließ, sondern auf eine Gelegenheit wartete, »die Größeren jener Region« zu demütigen, wie es Brunos Buch vom Sachsenkrieg berichtet. ${ }^{56}$

Die jedoch distanzierten sich vehement von der Zerstörung der Harzburg, wie Lampert von Hersfeld zum Jahre 1075 berichtet, sie könnten ihre Unschuld belegen, sie würden die Burg und die Kirche prächtiger aufbauen, als sie vorher gewesen, schließlich sei »das törichte Volk, vom bösen Geist angestachelt, in frevelhaftem Unterfangen « verantwortlich. ${ }^{57}$

Übereinstimmend werden in den zeitnahen sachsenfreundlichen Quellen mehrere Gründe für den Konflikt hervorgehoben. Der Burgenbau und Charakter Heinrichs IV., ${ }^{58}$ die "Bedrückungen « der Thüringer als Teil der Sachsen, die Konflikte Heinrichs IV. mit der Kirche und Papst Gregor VII. sowie, als wichtigstes Argument, die als bedroht empfundene Freiheit der Sachsen: Heinrich hätte gar die Ausrottung oder mindestens die Versklavung der Sachsen angestrebt, da deren freiheitsliebender »Charakter « seinen Zielen zuwiderliefe.

Alle in den Quellen der sächsischen Parteiungen genannten Aspekte sind, mit Ausnahme der geplanten Ausrottung, vermutlich subjektiv gegeben. Allerdings nur, wenn die sächsische Selbstsicht als legitim angesehen wird. In jedem Falle kann jenseits aller Bewertung festgestellt werden, dass in der zweiten Hälfte des I I. Jahrhunderts ein Bewusstsein der oppositionellen Kreise der Sachsen konstruiert wurde, das auf einem durchaus hohen Niveau operierte - man denke an die Reflexion über die Entbindung vom Eid oder die versuchte Einordnung der sächsischen Aufstandsbewegung in die aktuelle Debatte der Kirchenreformer und den Streit mit Gregor VII.

Dieser allerdings, dessen Briefe als Rechtfertigung der sächsischen Opposition in Brunos Buch vom Sachsenkrieg inseriert wurden, hat sich seinerseits nie zustimmend zu den Aufständen geäußert, sondern gar von der superbia Saxonum vobis iniuste resistentium in einem Brief vom September I075 (III/7) an den Salier gesprochen. ${ }^{59}$ Darüber hinaus lässt sich jedoch feststellen, dass offenbar die Auseinandersetzungen zwischen Sacerdotium und Imperium, der Kampf um das Recht der Investitur sowie die Diskussionen im christlichen Europa durchaus von der den Widerstand der Sachsen begleitenden Geschichtsschreibung wahrgenom-

Sachsen, Zitat auf 258. Sächsische Dinge betreffen die Briefe I/39 vom 20. Dezember 1073 [in der Edition irrtümlich: I037] (fordert die Sachsen zur Waffenruhe gegen Heinrich IV. auf, wie er auch diesen ermahnt habe), 6I f., sowie VIII/23 (S. 565 ff.), in dem auf Osnabrücker Urkundenfälschungen aufbauend von einer Übertragung Sachsens an den Papst durch Karl den Großen (Idem vero magnus imperator Saxoniam obtulit beato Petro, 566) die Rede ist. Vgl. Gerd Althoff, Heinrich IV., Darmstadt 2006, 205-209, zu dem Recht der Sachsen in den 8 oer Jahren des I I. Jahrhunderts und Otтo-Hubert Kost, Das östliche Niedersachsen im Investiturstreit. Studien zu Brunos Buch vom Sachsenkrieg, Göttingen I962, I $46 \mathrm{ff}$. zum ambivalenten Verhältnis der Sachsen zum Papst. 
men und auf die sächsischen Verhältnisse übertragen wurden, auch wenn hier das Verhältnis von Glauben und Recht, von Kirche und Welt, freilich nicht ausgreifend untersucht werden kann. ${ }^{60}$

Zugleich ist ein Zusammenhang zwischen der Entwicklung der Idee einer weitergehenden sächsischen Freiheit und der einer libertas ecclesiae zu erkennen. Dies bedarf weiterer Überlegungen, auf den ersten Blick jedoch dürften bei der vergleichenden Analyse des Rückbezuges auf einen Raum die größten Übereinstimmungen in der Argumentation sowohl der kirchlichen wie auch der weltlichen Seite zu finden sein.

\section{Zusammenfassung}

Es konnte gezeigt werden, dass sich im Laufe des 9. bis I I. Jahrhunderts in Sachsen ein an der Historiographie und an den Ereignissen erkennbares Bewusstsein der Sachsen entwickelt hat, das sich zunächst auf Menschen und später auch auf den Raum bezog. In der Stauferzeit erscheint dann der Begriff »Patria « vollends politisiert und auf das »Vaterland « bezogen, wie Eichenberger summiert. ${ }^{6 r}$

Bei Einhards Vita Karls des Großen steht die Unterwerfung und die Christianisierung der Sachsen im Vordergrund, der Poeta Saxo erweitert dies am Ende des 9. Jahrhunderts um die Garantie beziehungsweise Konzedierung oder gar den Erlass sächsischer Rechte. Im Io. Jahrhundert, während der Herrschaft Ottos I., legt Widukind von Corvey den Schwerpunkt auf die heilsgeschichtliche Notwendigkeit der sächsischen Niederlage und Bekehrung als Voraussetzung für einen erneuten Zusammenschluss auf gleicher Augenhöhe von Sachsen und Franken, der allerdings eine gewisse Zeit brauchte, wie Widukinds Halbsatz: »wie wir jetzt sehen « belegt. ${ }^{62}$ Hier wird schon früh der wesentliche Faktor »Zeit« von einem Beteiligten in den Diskurs eingeführt, der sich in der gestreckten Entwicklung eines personen- und raumbezogenen Gedankenmodells widerspiegelt.

In der ersten Hälfte des I I. Jahrhunderts schließlich ist die sächsische Position im Reichsverband so stark, dass sie Wahlen fernbleiben und die Elekten I002 und I024 sowie wohl auch I028 zur Reise in ihren Raum zwingen können. In der zweiten Hälfte desselben Jahrhunderts führt diese Stellung gar zu einem Aufstand

60 Vgl. auch die Überlegungen von

EICHenberger, Patria (Fn. I7) I68-I73.

6I Ebenda I73-I 82 .

$62[\ldots]$ veluti modo videmus $[\ldots]$.

Widukind von Corvey I/I 5

(Fn. 4). 
mit militärischen Auseinandersetzungen über mehrere Jahre regional zwar begrenzt, in der Historiographie aber terminologisch auf ganz Sachsen bezogen.

In einer staatenlosen, mithin: verfassungslosen Zeit ist dies ein Hinweis auf räumliche Verdichtung hin zu den erst später entstehenden Territorien. Eine Herausforderung für das Königtum, auf die zumindest Heinrich IV. nicht angemessen zu reagieren wusste, und - so soll abgeschlossen werden - vermutlich auch kein deutscher Herrscher nach ihm. Auf der anderen Seite wäre wohl ebenfalls zu konstatieren, dass bereits vor Heinrich IV. kein ostfränkisch-deutscher König in der Lage oder willens war, die Wurzeln der hier dargelegten Entwicklung auszutrocknen oder andere Früchte zu züchten.

Letztlich ist es die Zeit, die die Entwicklungen rechtfertigt. Sie ist die Voraussetzung für die von Widukind als abgeschlossen und beim zuvor schreibenden Poeta Saxo als intendiert geschilderte Integration der Sachsen in das fränkische »Reichsvolk «. Sie erlaubt es den Sachsen, nach hundertiähriger Ottonenherrschaft zu denken, sie seien »das wahre $>$ Reichsvolk $«$ «, ${ }^{63}$ und Otto von Northeim, von »alten " Freiheiten zu sprechen. Sie begründet durch Wiederholung überlieferter Verhaltensweisen der verschiedenen deutschen Könige im Umgang mit den Sachsen, dass von Traditionen gesprochen werden kann. ${ }^{64}$ Das alles natürlich nur, weil es stets Menschen gab, die sich mit der prägenden, argumentativen Kraft der Zeit auseinandersetzten und halfen, ihre Wirkung sichtbar zu machen in den Diskursen ihrer jeweiligen Lebenswirklichkeit. Nicht alles dabei sind Inventionen, Konstruktionen und Rückbezüge. Schließlich galt es, wie im hier kursorisch behandelten Fall, Vergangenheit, Gegenwart und Zukunft der Sachsen, ihres Rechtes und Raumes zu interpretieren und zu sichern.

Caspar Ehlers

63 Vgl. BeCHER, Volksbildung (Fn. I I) 83 f., Zitat 84 .

64 Vgl. RogGe, Könige (Fn. 32) 48, zu den Umständen der sächsischen

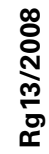

Wahl Wilhelms von Holland I 252, ein Vorgang, der, so Rogge, "an das Erscheinen von König Heinrich II. I002 bei den Sachsen in Merseburg « erinnere. Schon I 96 begann der Widerstand der Fürsten gegen den so genannten »Erbreichsplan « Heinrichs VI. auf einer Versammlung in Merseburg, vgl. Ludwig Vones, Confirmatio Imperii et Regni. Erbkaisertum, Erbreichsplan und Erbmonarchie in den politischen Zielvorstellungen der letzten Jahre Kaiser Heinrichs VI, in: Stauferreich im Wandel. Ordnungsvorstellungen und Politik in der Zeit Friedrich Barbarossas, hg. von STEFAN WeinfurTer, Stuttgart 2002, 324 f., was vermutlich durch die Königsferne Sachsens seit dem ausgehenden I3. Jahrhundert begründet werden kann. 